ISSN 1561-2430 (Print)

ISSN 2524-2415 (Online)

УДК 621.315 .592

Поступила в редакцию 03.09.2020

https://doi.org/10.29235/1561-2430-2020-56-4-480-487

Received 03.09.2020

\author{
Е. А. Толкачева ${ }^{1}$, В. П. Маркевич ${ }^{2}$, Л. И. Мурин ${ }^{1}$ \\ ${ }^{1}$ Научно-практический центр Национальной академии наук Беларуси по материаловедению, \\ Минск, Беларусь \\ ${ }^{2}$ Университет г. Манчестер, Манчестер, Великобритания

\section{ЛОКАЛЬНЫЕ КОЛЕБАТЕЛЬНЫЕ МОДЫ ВАКАНСИОННО-КИСЛОРОДНЫХ КОМПЛЕКСОВ В КРИСТАЛЛИЧЕСКОМ КРЕМНИИ ПРИ КОМНАТНОЙ ТЕМПЕРАТУРЕ}

\begin{abstract}
Аннотация. Изотопный состав природного кремния $\left({ }^{28} \mathrm{Si}(92,23 \%),{ }^{29} \mathrm{Si}(4,68 \%)\right.$ и $\left.{ }^{30} \mathrm{Si}(3,09 \%)\right)$ оказывает заметное влияние на форму полос ИК-поглощения, обусловленных примесными атомами кислорода. В настоящей работе предпринята попытка определить положение локальных колебательных мод (ЛКМ), обусловленных квазимолекулами ${ }^{28} \mathrm{Si}_{-}{ }^{16} \mathrm{O}_{S^{-}}{ }^{29} \mathrm{Si}$ и ${ }^{28} \mathrm{Si}_{-}{ }^{16} \mathrm{O}_{S^{-}}{ }^{30} \mathrm{Si}\left(\mathrm{O}_{S}-\right.$ атом кислорода в узле решетки), для спектров поглощения, измеренных при комнатной температуре. Проведена оценка изотопических сдвигов соответствующих мод путем подгонки формы полосы поглощения для комплекса вакансия-кислород (А-центр) в облученных кристаллах $\mathrm{Si}$. Изотопические сдвиги ЛКМ равны $2,2 \pm 0,25 \mathrm{~cm}^{-1}$ для ${ }^{28} \mathrm{Si}^{16}{ }^{16} \mathrm{O}_{S^{-}}{ }^{29} \mathrm{Si}$ и $4,3 \pm 0,9 \mathrm{~cm}^{-1}$ для ${ }^{28} \mathrm{Si}^{1-}{ }^{16} \mathrm{O}_{S^{-}}{ }^{30} \mathrm{Si}$ по отношению к полосе ${ }^{28} \mathrm{Si}^{-}{ }^{16} \mathrm{O}_{S^{-}}{ }^{28} \mathrm{Si}$, а полуширина полосы поглощения А-центра $\left({ }^{28} \mathrm{Si}-{ }^{16} \mathrm{O}_{S^{-}}{ }^{28} \mathrm{Si}\right)$ составляет $5,3 \pm 0,25 \mathrm{~cm}^{-1}$. Методом ИК-спектроскопии установлено, что в температурном интервале отжига дивакансий $\left(200-275{ }^{\circ} \mathrm{C}\right)$ в облученных кислородсодержащих кристаллах кремния имеет место формирование двух полос поглощения с максимумами у 825,8 и $839,2 \mathrm{~cm}^{-1}$. Комплексу дивакансия-кислород $\mathrm{V}_{2} \mathrm{O}$, образующемуся путем захвата подвижных $\mathrm{V}_{2}$ междоузельными атомами кислорода $\mathrm{O}_{i}$, приписана полоса у $825,8 \mathrm{~cm}^{-1}$. Относительная интенсивность полосы у $839,2 \mathrm{~cm}^{-1}$ существенно увеличивается в образцах, облученных нейтронами, по сравнению с образцами, облученными электронами. Сделано заключение, что эта полоса связана с комплексом тривакансия-кислород $\mathrm{V}_{3} \mathrm{O}$, образующимся путем захвата подвижных $\mathrm{V}_{3}$ атомами $\mathrm{O}_{i}$.

Ключевые слова: локальные колебательные моды, кремний, ИК-поглощение, изотопический сдвиг, изотопный состав

Для цитирования. Толкачева, Е. А. Локальные колебательные моды вакансионно-кислородных комплексов в кристаллическом кремнии при комнатной температуре / Е. А. Толкачева, В. П. Маркевич, Л. И. Мурин // Вес. Нац. акад. навук Беларусі. Сер. фіз.-мат. навук. - 2020. - Т. 56, № 4. - С. 480-487. https://doi.org/10.29235/1561-2430-2020-56$4-480-487$
\end{abstract}

\author{
Ekaterina A. Tolkacheva ${ }^{1}$, Vladimir P. Markevich ${ }^{2}$, Leonid I. Murin ${ }^{1}$ \\ ${ }^{I}$ Scientific and Practical Materials Research Center of the National Academy of Sciences of Belarus, Minsk, Belarus \\ ${ }^{2}$ The University of Manchester, Manchester, Great Britain
}

\title{
LOCAL VIBRATIONAL MODES OF VACANCY-OXYGEN-RELATED COMPLEXES AT ROOM TEMPERATURE
}

\begin{abstract}
The isotopic content of natural silicon $\left({ }^{28} \mathrm{Si}(92.23 \%),{ }^{29} \mathrm{Si}(4.68 \%)\right.$ и $\left.{ }^{30} \mathrm{Si}(3.09 \%)\right)$ affects noticeably the shape of IR absorption bands related to the oxygen impurity atoms. In the present work an attempt is undertaken to determine the

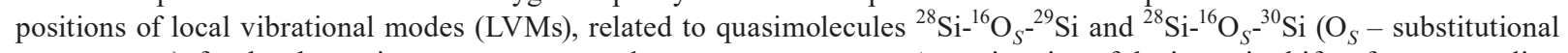
oxygen atom), for the absorption spectra measured at room temperature. An estimation of the isotopic shifts of corresponding modes is done by fitting the shape of the experimentally measured absorption band related to the vacancy-oxygen center in irradiated Si crystals. The LVM isotope shifts are found to be equal $2,2 \pm 0.25 \mathrm{~cm}^{-1}$ for ${ }^{28} \mathrm{Si}^{16} \mathrm{O}_{S^{-}}{ }^{29} \mathrm{Si}$ and $4,3 \pm 0,9 \mathrm{~cm}^{-1}$ for ${ }^{28} \mathrm{Si}^{16} \mathrm{O}_{S^{-}}{ }^{30} \mathrm{Si}$ in relation to the basic band due to ${ }^{28} \mathrm{Si}^{-16} \mathrm{O}_{S^{-}}{ }^{28} \mathrm{Si}$, and the full width at half maximum of the A-center absorption band $\left({ }^{28} \mathrm{Si}^{-16} \mathrm{O}_{\mathrm{S}^{-}}{ }^{28} \mathrm{Si}\right)$ is $5,3 \pm 0.25 \mathrm{~cm}^{-1}$. By means of infrared absorption spectroscopy a clear correlation between the disappearance of the divacancy $\left(\mathrm{V}_{2}\right)$ in the temperature range $200-275{ }^{\circ} \mathrm{C}$ and appearance of two absorption bands with their maxima at 825.8 and $839.2 \mathrm{~cm}^{-1}$ in irradiated oxygen-rich Si crystals is found. The band positioned at $825.8 \mathrm{~cm}^{-1}$ is assigned to a divacancy-oxygen defect $\mathrm{V}_{2} \mathrm{O}$ formed via an interaction of mobile $\mathrm{V}_{2}$ with interstitial oxygen $\left(\mathrm{O}_{i}\right)$ atoms. The $839.2 \mathrm{~cm}^{-1}$ band is much more pronounced in neutron irradiated samples as compared to samples irradiated with electrons. We argue that it is related to a trivacancy-oxygen defect $\left(\mathrm{V}_{3} \mathrm{O}\right)$ formed via an interaction of mobile $\mathrm{V}_{3}$ with $\mathrm{O}_{i}$ atoms.
\end{abstract}

Keywords: local vibrational mode; silicon; infra-red absorption; isotopic shift; isotopic content

(C) Толкачева Е. А., Маркевич В. П., Мурин Л. И., 2020 
For citation. Tolkacheva E. A., Markevich V. P., Murin L. I. Local vibrational modes of vacancy-oxygen-related complexes at room temperature. Vestsi Natsyianal'nai akademii navuk Belarusi. Seryia fizika-matematychnykh navuk = Proceedings of the National Academy of Sciences of Belarus. Physics and Mathematics series, 2020, vol. 56, no. 4, pp. 480487 (in Russian). https://doi.org/10.29235/1561-2430-2020-56-4-480-487

Введение. Наиболее важной технологической примесью (после основных легирующих) в кремнии является кислород [1]. Высокотемпературные обработки, а также облучение высокоэнергетическими частицами кислородсодержащих кристаллов $\mathrm{Si}$ и их последующий отжиг приводят к образованию различного рода электрически и оптически активных центров, включающих в свой состав примесные атомы кислорода [1-4]. Из них особый интерес представляют вакансионно-кислородные комплексы. Благодаря наличию глубоких уровней и высокой термической стабильности такие комплексы могут служить эффективными центрами рекомбинации неосновных носителей заряда и следует ожидать их применения в радиационной технологии изготовления кремниевых быстродействующих приборов [5]. Кроме того, предполагается [4], что некоторые из этих комплексов являются центрами зарождения кислородных преципитатов, формирование которых используется в современной технологии кремниевых приборов и интегральных микросхем для геттерирования нежелательных примесей (Cu, Fe и т. д.). В связи с этим уже в течение ряда лет интенсивно изучаются механизмы формирования и свойства кислородсодержащих центров в кремнии.

Одной из наиболее успешно применяющихся методик исследования кислородсодержащих центров в кремнии является спектроскопия локальных колебательных мод (ЛКМ) [1-4, 6, 7]. В частности, методом ИК-поглощения была проведена идентификация кислородных димеров и ряда вакансионно-кислородных комплексов $\mathrm{VO}_{n}(n \geq 1)[2,3]$. В то же время имеющиеся в литературе данные о локальных колебательных модах так называемых мультивакансионных кислородсодержащих комплексов $\mathrm{V}_{n} \mathrm{O}(n \geq 2)$ весьма неоднозначны $[3,6,8]$. В первую очередь это связано с тем, что комплексы $\mathrm{V}_{n} \mathrm{O}(n \geq 2)$ содержат такую же структурную единицу $\mathrm{Si}-\mathrm{O}-\mathrm{Si}$, как и комплекс вакансия-кислород (VO или А-центр) и частоты валентных колебаний кислорода очень близки для всех центров. На эксперименте это проявляется в том, что даже в спектрах, измеренных при низкой температуре (НT), имеет место перекрытие полос поглощения, обусловленных разными комплексами [6]. При этом полоса поглощения, обусловленная VO, как правило, доминирует. Только тщательный анализ формы полос, обусловленных $\mathrm{V}_{2} \mathrm{O}$ и $\mathrm{V}_{3} \mathrm{O}$, с учетом изотопного состава кристаллов $\mathrm{Si}$ (природный кремний состоит из изотопов ${ }^{28} \mathrm{Si}(92,23 \%),{ }^{29} \mathrm{Si}(4,68 \%)$ и ${ }^{30} \mathrm{Si}(3,09$ \%) [7]), позволил провести идентификацию ЛКМ соответствующих центров [6].

Ситуация является еще более сложной при интерпретации спектров поглощения, измеренных при комнатной температуре (КТ). В этом случае полуширины полос, обусловленных атомами кислорода, находящимися в вакансии (дивакансии, тривакансии), в несколько раз превышают как аналогичные величины, полученные из анализа спектров, измеренных при низкой температуре, так и значения изотопических сдвигов полос комплексов $\mathrm{V}_{n} \mathrm{O}$, когда один из атомов ${ }^{28} \mathrm{Si}$ в структуре $\mathrm{Si}-\mathrm{O}-\mathrm{Si}$ заменен атомами ${ }^{29} \mathrm{Si}$ или ${ }^{30} \mathrm{Si}$, либо оба атома ${ }^{28} \mathrm{Si}$ заменены изотопами (см. далее). Как результат, в спектрах поглощения, измеренных при комнатной температуре, имеет место большее перекрытие близкорасположенных полос поглощения, что в свою очередь может снизить достоверность и однозначность результатов анализа (подгонки) соответствующих спектров.

Несмотря на очевидные преимущества ИК-измерений колебательных спектров при низкой температуре, в ряде случаев получить нужную информацию из таких измерений не представляется возможным. Это относится в первую очередь к образцам $\mathrm{Si}$, в $\mathrm{HT}$ спектрах которых наблюдается сильное поглощение, обусловленное электронными переходами на водородо- и гелиоподобных центрах [9]. Кроме того, в ряде случаев у исследователей просто отсутствует оборудование, необходимое для проведения НТ измерений спектров ИК-поглощения. В связи с вышеизложенным значительный интерес представляет разработка методики детального анализа спектров ИК-поглощения, измеренных при комнатной температуре, для облученных и отожженных кристаллов кремния. Очевидно, что для анализа сложных спектров, обусловленных суперпозицией ЛКМ вакансионно-кислородных комплексов в природном кремнии, требуется знание величин 
кремниевых изотопических сдвигов для валентного колебания комплекса VO (А-центра) при КТ. Определение значений таких сдвигов и являлось одной из целей настоящей работы. Полученные данные были использованы затем для идентификации ЛКМ комплексов $\mathrm{V}_{2} \mathrm{O}$ и $\mathrm{V}_{3} \mathrm{O}$ при комнатной температуре.

Методика исследований. Исследовались кристаллы кремния $n$-типа, полученные методом Чохральского $(\mathrm{Cz}-\mathrm{Si})$. Концентрации $\mathrm{O}_{i}\left(\left[\mathrm{O}_{i}\right]=(8-11) \cdot 10^{17} \mathrm{~cm}^{-3}\right)$ и углерода замещения $\left(\left[\mathrm{C}_{s}\right]=(0,5-7) \cdot 10^{16} \mathrm{~cm}^{-3}\right)$ определялись по результатам измерений интенсивности хорошо известных полос поглощения 1107 и $605 \mathrm{~cm}^{-1}$ с использованием калибровочных коэффициентов $3,14 \cdot 10^{17}$ и $0,94 \cdot 10^{17} \mathrm{~cm}^{-2}$ для кислорода и углерода соответственно [2]. Образцы полировались с двух сторон и имели размеры $10 \times 6 \times 3$ или $10 \times 6 \times 5$ мм $^{3}$.

Облучение высокоэнергетическими протонами $(E=24$ ГэВ), быстрыми электронами $(E=2,6$ и $10 \mathrm{MэB)} \mathrm{и} \mathrm{нейтронами} \mathrm{проводилось} \mathrm{при} \mathrm{комнатной} \mathrm{температуре}(\leq 350$ К) различными дозами от $1 \cdot 10^{16}$ до $1 \cdot 10^{18} \mathrm{~cm}^{-2}$, и образцы обычно хранились при комнатной температуре в течение нескольких недель перед измерениями. Образцы были подвергнуты 30 -минутному изохронному отжигу в диапазоне $75-400{ }^{\circ} \mathrm{C}$ с шагом $25^{\circ} \mathrm{C}$. Спектры ИК-поглощения измерялись на Фурье-спектрометре Bruker 113v при комнатной температуре. Спектральное разрешение составляло $1,0 \mathrm{~cm}^{-1}$.

Результаты и их обсуждение. Мы попытались оценить изотопические сдвиги соответствующих мод полуэмпирическим путем, т. е. путем подгонки формы полосы поглощения VO, используя функции Лоренца с фиксированными соотношениями амплитуд, соответствующими относительным концентрациям различных изотопных комбинаций Si-O-Si в природном кремнии, аналогично тому, как это было сделано в работе [7]. При оценке изотопических сдвигов соответствующих мод полуэмпирическим путем относительная интенсивность для основной полосы поглощения ${ }^{28} \mathrm{Si}^{16} \mathrm{O}_{S^{-}}{ }^{28} \mathrm{Si}$ принималась равной 1 , суммарная интенсивность полос поглощения для изотопных модификаций ${ }^{28} \mathrm{Si}^{-16} \mathrm{O}_{S^{-}}{ }^{30} \mathrm{Si}$ и ${ }^{29} \mathrm{Si}^{16} \mathrm{O}_{\mathrm{S}^{-}}{ }^{29} \mathrm{Si}$ составила 0,0696 , для ${ }^{28} \mathrm{Si}^{16} \mathrm{O}_{S^{-}}{ }^{29} \mathrm{Si}-0,1015$. Подгоночными параметрами являлись положения локальных колебательных мод и значения полуширин соответствующих полос, которые задавались одинаковыми для всех изотопных комбинаций. Было проанализировано большое количество экспериментально измеренных спектров поглощения (в качестве примера на рис. 1 показан один из таких спектров). Характеристики исследуемых образцов представлены в табл. 1, полученные значения положения основной полосы А-центра (комбинация $\left.{ }^{28} \mathrm{Si}^{16} \mathrm{O}_{\mathrm{S}^{-}}{ }^{28} \mathrm{Si}\right)$, изотопических сдвигов и полуширины данной полосы приведены в табл. 2.

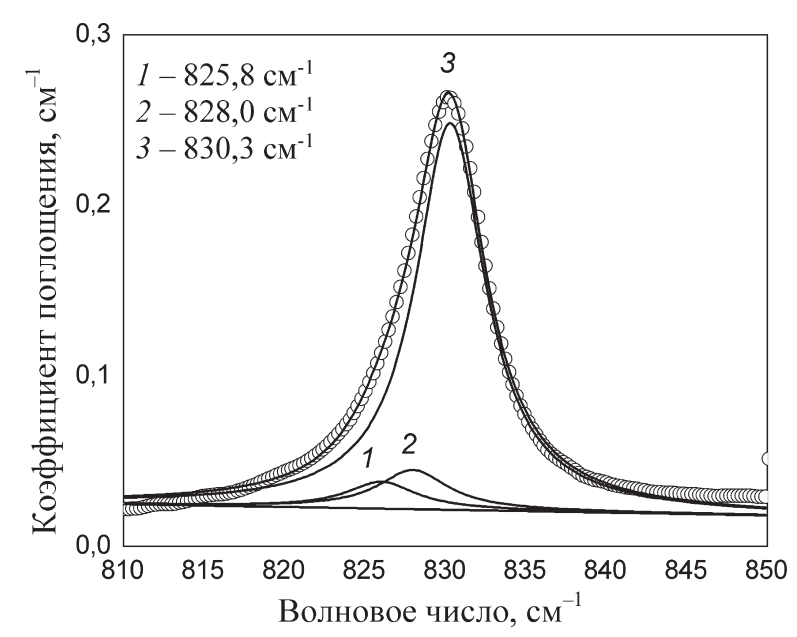

Рис. 1. Фрагмент спектра поглощения, измеренного при комнатной температуре для образца $\mathrm{Cz}-\mathrm{Si}\left(\left[\mathrm{O}_{i}\right]=1,3 \cdot 10^{18}\right.$, $\left.\left[\mathrm{C}_{s}\right]=5 \cdot 10^{15},[\mathrm{P}]=8 \cdot 10^{13} \mathrm{~cm}^{-3}\right)$, облученного электронами $\left(E=10 \mathrm{MэB}, F=3 \cdot 10^{17} \mathrm{~cm}^{-2}\right)$. Сплошные линии - подгонка с использованием функции Лоренца после коррекции базовой линии

Fig. 1. Fragment of the absorption spectrum measured at room temperature for a $\mathrm{Cz}-\mathrm{Si}$ sample $\mathrm{Cz}-\mathrm{Si}\left(\left[\mathrm{O}_{i}\right]=1,3 \cdot 10^{18}\right.$, $\left.\left[\mathrm{C}_{s}\right]=5 \cdot 10^{15},[\mathrm{P}]=8 \cdot 10^{13} \mathrm{~cm}^{-3}\right)$ irradiated with electrons $\left(E=10 \mathrm{M \ni B}, F=3 \cdot 10^{17} \mathrm{~cm}^{-2}\right)$. Solid lines - 
Полученные средние значения изотопических сдвигов локальных колебательных мод оказались равными $2,21 \mathrm{~cm}^{-1}$ для ${ }^{28} \mathrm{Si}_{-}{ }^{16} \mathrm{O}-{ }^{29} \mathrm{Si}$ и $4,29 \mathrm{~cm}^{-1}$ для ${ }^{28} \mathrm{Si}-{ }^{16} \mathrm{O}-{ }^{30} \mathrm{Si}$, что заметно превышает соответствующие величины $\left(1,51\right.$ и 2,92 $\left.\mathrm{cm}^{-1}\right)$, полученные из анализа низкотемпературных спектров. В данный момент причины таких отличий не совсем понятны. Следует отметить, что максимум поглощения для основной комбинации ${ }^{28} \mathrm{Si}-{ }^{16} \mathrm{O}-{ }^{28} \mathrm{Si}$ при комнатной температуре расположен у 830,3 $\mathrm{cm}^{-1}$, т. е. смещен на $5,5 \mathrm{~cm}^{-1}$ в область более низких частот от своего положения у $835,8 \mathrm{~cm}^{-1}$ при низкой температуре. При этом полуширина соответствующей полосы выросла от 2,34 $\mathrm{cm}^{-1}$ [7] при НТ до 5,31 $\mathrm{cm}^{-1}$ при КТ.

Таблииа 1. Характеристики исследуемых образцов

Table 1. Characteristics of the studied samples

\begin{tabular}{|c|c|c|c|c|c|c|}
\hline Материал & $\begin{array}{c}\text { Тип облучения, } \\
\text { доза, } \text { см }^{-2}\end{array}$ & $\begin{array}{l}\text { Энергия } \\
\text { облучения }\end{array}$ & Отжиг, ${ }^{\circ} \mathrm{C}$ & \begin{tabular}{c|} 
Концентрация \\
легирующей примеси, см ${ }^{-3}$
\end{tabular} & $\begin{array}{c}\text { Концентрации междоузель- } \\
\text { нгго кислорода }\left[\mathrm{O}_{i}\right], \mathrm{cm}^{-3}\end{array}$ & $\begin{array}{c}\text { Концентрации углерода } \\
\text { замещения }\left[\mathrm{C}_{s}\right], \mathrm{cm}^{-3}\end{array}$ \\
\hline \multirow{2}{*}{ WL-6 } & \multirow{2}{*}{$\begin{array}{c}\text { Электроны, } \\
3 \cdot 10^{17}\end{array}$} & $10 \cdot \mathrm{MэB}$ & 100 & {$[\mathrm{P}]=8 \cdot 10^{13}$} & $1,3 \cdot 10^{18}$ & $5 \cdot 10^{15}$ \\
\hline & & $10 \cdot \mathrm{MэB}$ & 150 & {$[\mathrm{P}]=8 \cdot 10^{13}$} & $1,3 \cdot 10^{18}$ & $5 \cdot 10^{15}$ \\
\hline 3-J4 & $\begin{array}{c}\text { Протоны, } \\
1 \cdot 10^{16}\end{array}$ & $24 \cdot \Gamma э \mathrm{~B}$ & - & {$[\mathrm{P}]=1 \cdot 10^{14}$} & $1,1 \cdot 10^{18}$ & $\sim 10^{15}$ \\
\hline \multirow{3}{*}{ Val-2-1 } & \multirow{3}{*}{$\begin{array}{c}\text { Электроны, } \\
3 \cdot 10^{17}\end{array}$} & $10 \cdot \mathrm{Mэ}$ & - & {$[\mathrm{P}]=1 \cdot 10^{14}$} & $8,3 \cdot 10^{14}$ & $7 \cdot 10^{16}$ \\
\hline & & $10 \cdot \mathrm{MэB}$ & 100 & {$[\mathrm{P}]=1 \cdot 10^{14}$} & $8,3 \cdot 10^{14}$ & $7 \cdot 10^{16}$ \\
\hline & & $10 \cdot \mathrm{MэB}$ & 150 & {$[\mathrm{P}]=1 \cdot 10^{14}$} & $8,3 \cdot 10^{14}$ & $7 \cdot 10^{16}$ \\
\hline $\mathrm{W}-44$ & $\begin{array}{c}\text { Электроны, } \\
1 \cdot 10^{18}\end{array}$ & $2 \cdot \mathrm{MэB}$ & - & {$[\mathrm{P}]=8 \cdot 10^{13}$} & $1,2 \cdot 10^{18}$ & $\sim 5 \cdot 10^{15}$ \\
\hline W027 & $\begin{array}{c}\text { Электроны, } \\
1 \cdot 10^{17}\end{array}$ & $2 \cdot \mathrm{MэB}$ & - & {$[\mathrm{P}]=8 \cdot 10^{13}$} & $1,2 \cdot 10^{18}$ & $\leq 1 \cdot 10^{15}$ \\
\hline $1-\mathrm{I} 4$ & $\begin{array}{c}\text { Протоны, } \\
1 \cdot 10^{16}\end{array}$ & $24 \cdot \Gamma э \mathrm{~B}$ & - & {$[\mathrm{P}]=1 \cdot 10^{14}$} & $9 \cdot 10^{17}$ & $7 \cdot 10^{16}$ \\
\hline
\end{tabular}

Таблица 2. Характеристики колебательных полос для изотопических модификаций квазимолекулы $\mathrm{Si}^{16} \mathrm{O}_{s}-\mathrm{Si}$ для центра VO, определенные путем подгонки лоренцианами (комнатные температуры)

Table 2. Characteristics of vibrational bands for isotopic modifications of the quasimolecule $\mathrm{Si}^{-16} \mathrm{O}_{S}-\mathrm{Si}$ for the VO center, determined by Lorentzians fitting (room temperatures)

\begin{tabular}{|c|c|c|c|c|c|}
\hline Материал & Отжиг, ${ }^{\circ} \mathrm{C}$ & $\begin{array}{c}{ }^{28} \mathrm{Si}-\mathrm{O}-{ }^{28} \mathrm{Si} \\
\text { Положение, } \mathrm{cm}^{-1}\end{array}$ & $\begin{array}{l}{ }^{29} \mathrm{Si}-\mathrm{O}_{-}{ }^{28} \mathrm{Si} \\
\text { Сдвиг, см }{ }^{-1}\end{array}$ & $\begin{array}{l}{ }^{30} \mathrm{Si}-\mathrm{O}-{ }^{28} \mathrm{Si} \\
\text { Сдвиг, см }{ }^{-1}\end{array}$ & Полуширина, cм $^{-1}$ \\
\hline \multirow{2}{*}{ WL-6 } & - & 830,29 & 2,3 & 4,53 & 5,27 \\
\hline & 150 & 830,29 & 2,27 & 4,48 & 5,30 \\
\hline $3-\mathrm{J} 4$ & - & 830,34 & 1,99 & 3,83 & 5,50 \\
\hline \multirow{3}{*}{ Val-2-1 } & - & 830,29 & 2,09 & 4,17 & 5,33 \\
\hline & 100 & 830,31 & 2,23 & 4,34 & 5,30 \\
\hline & 150 & 830,30 & 2,35 & 4,59 & 5,36 \\
\hline $\mathrm{W}-44$ & - & 830,32 & 2,4 & 4,32 & 5,26 \\
\hline W027 & - & 830,3 & 2,07 & 4,05 & 5,18 \\
\hline $1-\mathrm{I} 4$ & - & 830,35 & 2,47 & 3,39 & 5,04 \\
\hline \multicolumn{2}{|c|}{ Среднее значение } & 830,3 & 2,21 & 4,29 & 5,31 \\
\hline
\end{tabular}

Полученные значения основных оптических характеристик А-центров позволили провести идентификацию ЛКМ комплексов $\mathrm{V}_{2} \mathrm{O}$ и $\mathrm{V}_{3} \mathrm{O}$ при комнатной температуре.

Локальные колебательные моды комплексов дивакансия-кислород и тривакансия-кислород при комнатной температуре. Очевидно, что в процессе облучения при $T \sim 300 \mathrm{~K}$ дефекты $\mathrm{V}_{n} \mathrm{O}$ в кремнии могут возникнуть путем последовательного захвата подвижных вакансий ловушками $\mathrm{O}_{i}, \mathrm{VO}, \mathrm{V}_{2} \mathrm{O}$ и так далее, т. е. через реакции $\mathrm{V}+\mathrm{O}_{i} \Rightarrow \mathrm{VO}, \mathrm{V}+\mathrm{VO} \Rightarrow \mathrm{V}_{2} \mathrm{O}, \mathrm{V}+\mathrm{V}_{n} \mathrm{O} \Rightarrow \mathrm{V}_{n+1} \mathrm{O}$. Однако в $\mathrm{Cz}-\mathrm{Si}$, где концентрация кислорода $\sim 10^{18} \mathrm{~cm}^{-3}$, генерация $\mathrm{V}_{n} \mathrm{O}(n \geq 2)$ может быть эффективной только при очень больших дозах облучения, когда концентрация VO сопоставима с концентрацией $\mathrm{O}_{i}$. 

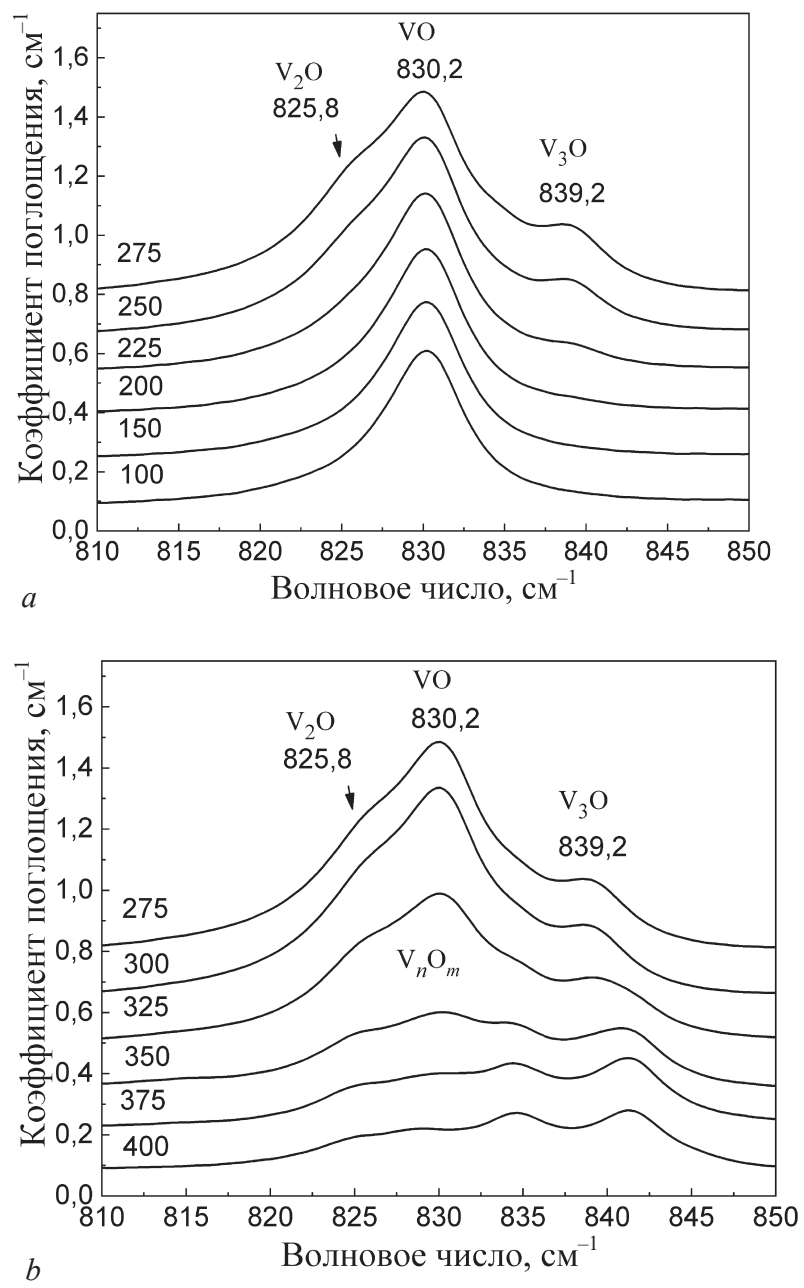

Рис. 2. Развитие спектра поглощения, измеренного при $T=300$ К для образца $\mathrm{Cz}-\mathrm{Si}\left(\left[\mathrm{O}_{i}\right]=8,3 \cdot 10^{17},\left[\mathrm{C}_{s}\right]=7 \cdot 10^{16},[\mathrm{P}]\right.$ $\left.=1 \cdot 10^{14} \mathrm{~cm}^{-3}\right)$, облученного нейтронами $\left(F=7,5 \cdot 10^{16} \mathrm{~cm}^{-2}\right)$, в процессе изохронного отжига $100-275^{\circ} \mathrm{C}(a) ;$ развитие спектров поглощения, измеренных при $T=300 \mathrm{~K}$ для образца $\mathrm{Cz}-\mathrm{Si}\left(\left[\mathrm{O}_{i}\right]=8,3 \cdot 10^{17},\left[\mathrm{C}_{s}\right]=7 \cdot 10^{16},[\mathrm{P}]=1 \cdot 10^{14} \mathrm{~cm}^{-3}\right)$, облученного нейтронами $\left(F=7,5 \cdot 10^{16} \mathrm{~cm}^{-2}\right)$, в процессе изохронного отжига $275-400{ }^{\circ} \mathrm{C}(b)$

Fig. 2. Development of the absorption spectrum measured at $T=300 \mathrm{~K}$ for the sample $\mathrm{Cz}-\mathrm{Si}\left(\left[\mathrm{O}_{i}\right]=8.3 \cdot 10^{17},\left[\mathrm{C}_{s}\right]=7 \cdot 10^{16}\right.$, $\left.[\mathrm{P}]=1 \cdot 10^{14} \mathrm{~cm}^{-3}\right)$, irradiated neutrons $\left(F=7,5 \cdot 10^{16} \mathrm{~cm}^{-2}\right)$, during isochronous annealing $100-275^{\circ} \mathrm{C}(a)$; development of absorption spectra measured at $T=300 \mathrm{~K}$ for the sample $\mathrm{Cz}-\mathrm{Si}\left(\left[\mathrm{O}_{i}\right]=8.3 \cdot 10^{17},\left[\mathrm{C}_{s}\right]=7 \cdot 10^{16},[\mathrm{P}]=1 \cdot 10^{14} \mathrm{~cm}^{-3}\right)$, irradiated neutrons $\left(F=7.5 \cdot 10^{16} \mathrm{~cm}^{-2}\right)$, during isochronous annealing $275-400^{\circ} \mathrm{C}$

При относительно небольших дозах, когда концентрация радиационных дефектов намного ниже концентрации $\mathrm{O}_{i}$, образование $\mathrm{V}_{2} \mathrm{O}$ незначительно даже в случае облучения нейтронами, и основное формирование комплексов $\mathrm{V}_{n} \mathrm{O}(n \geq 2)$ имеет место в процессе последующих отжигов облученных кристаллов кремния.

На рис. 2 показано развитие спектров поглощения в интервале волновых чисел 810-850 $\mathrm{cm}^{-1}$, обусловленных комплексами $\mathrm{V}_{n} \mathrm{O}$, в процессе изохронного отжига образца, облученного быстрыми нейтронами. В данном интервале волновых чисел наблюдается формирование и последующий отжиг ряда полос, обусловленных комплексами $\mathrm{V}_{n} \mathrm{O}_{m}$. Форма полос была проанализирована при помощи подгонки с учетом влияния изотопов кремния ${ }^{29} \mathrm{Si}$ и ${ }^{30} \mathrm{Si}$. При этом значения изотопических сдвигов и величины полуширин для всех центров задавались равными таковым, определенным нами для комплексов VO. После облучения в интервале волновых чисел $810-$ $850 \mathrm{~cm}^{-1}$ наблюдается только полоса А-центра (рис. 2, a). При температурах отжига выше $200{ }^{\circ} \mathrm{C}$ появляются дополнительные пики, связанные с вакансионно-кислородными комплексами $\mathrm{V}_{n} \mathrm{O}$, $n \geq 2$. Последующий отжиг (рис. $2, b$ ) при температурах выше $300{ }^{\circ} \mathrm{C}$ приводит к снижению 


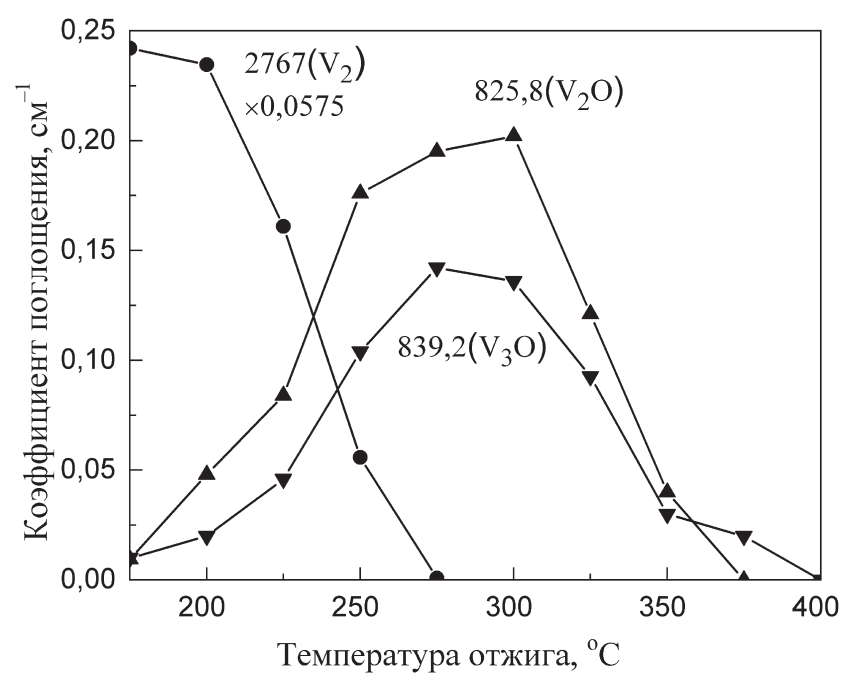

Рис. 3. Зависимости амплитуд полос поглощения 2767; 833,4 и 842,4 см ${ }^{-1}$, обусловленных комплексами $\mathrm{V}_{2}, \mathrm{~V}_{2} \mathrm{O}$ и $\mathrm{V}_{3} \mathrm{O}$, от температуры изохронного отжига

Fig. 3. Dependences of the amplitudes of the absorption bands at $2767,833.4$, and $842.4 \mathrm{~cm}^{-1}$, caused by complexes $\mathrm{V}_{2}, \mathrm{~V}_{2} \mathrm{O}$ and $\mathrm{V}_{3} \mathrm{O}$, on the temperature of isochronous annealing

интенсивности соответствующих пиков и появлению ряда новых, но уже менее интенсивных пиков, обусловленных, вероятнее всего, комплексами $\mathrm{V}_{n} \mathrm{O}_{m}(n, m \geq 2)$.

Дивакансии в кремнии подвижны при температурах выше $200^{\circ} \mathrm{C}$ и в кристаллах $\mathrm{Cz}-\mathrm{Si}$ междоузельный кислород является основной ловушкой подвижных $V_{2}[6]$, т. е. имеет место преобразование $\mathrm{V}_{2}$ в $\mathrm{V}_{2} \mathrm{O}$ через реакцию $\mathrm{V}_{2}+\mathrm{O}_{\mathrm{i}} \Rightarrow \mathrm{V}_{2} \mathrm{O}$. Отметим, что возможность такой реакции была недавно подтверждена в детальных исследованиях отжига дивакансий и тривакансий методом DLTS [10]. Появление новых дефектов после исчезновения $\mathrm{V}_{2}$ также ясно наблюдалось в наших исследованиях методом ИК-поглощения.

Отжиг дивакансии в образцах $\mathrm{Cz}-\mathrm{Si}$ сопровождается появлением новых полос поглощения. Самые интенсивные из них расположены у 825,8 и $839,2 \mathrm{~cm}^{-1}$. Исследования изохронного отжига показали, что не только процессы их формирования, но также и кинетика отжига очень подобны. Полосы 825,8 и 839,2 $\mathrm{cm}^{-1}$ исчезают одновременно после отжига в температурном диапазоне 300-375 ${ }^{\circ} \mathrm{C}$. На рис. 3 представлены зависимости амплитуд соответствующих полос от температуры изохронного отжига.

Можно предположить, что обе полосы связаны с одним и тем же дефектом, а именно $\mathrm{V}_{2} \mathrm{O}$, находящимся в различных конфигурациях. Однако есть факты, которые не подтверждают такое предположение. Было обнаружено, что отношение интенсивностей этих полос различно в образцах, облученных быстрыми электронами и нейтронами, т. е. зависит от вида облучения (энергии и массы частиц). Полоса $839,2 \mathrm{~cm}^{-1}$ является намного более интенсивной при нейтронном облучении. Это свидетельствует о том, что она возникает из более сложного дефекта, чем полоса $825,8 \mathrm{~cm}^{-1}$. Возможный кандидат - дефект $\mathrm{V}_{3} \mathrm{O}$. Вероятнее всего, тривакансии $\mathrm{V}_{3}$, образующиеся главным образом как первичные дефекты, обладают такой же миграционной способностью, как и $\mathrm{V}_{2}$, и $\mathrm{V}_{3} \mathrm{O}$ может образовываться через реакцию $\mathrm{V}_{3}+\mathrm{O}_{i} \Rightarrow \mathrm{V}_{3} \mathrm{O}$. Такое заключение хорошо согласуется с недавними результатами, полученными методом DLTS по отжигу тривакансий и их трансформации в комплексы $\mathrm{V}_{3} \mathrm{O}$ [10]. Эти данные также хорошо согласуются с данными для комплексов $\mathrm{V}_{2} \mathrm{O}$ и $\mathrm{V}_{3} \mathrm{O}$, полученными из измерений при низких температурах [6]. При изменении температуры измерения от низких к комнатным имеет место небольшое смещение пиков, принадлежащих дефектам $\mathrm{V}_{2} \mathrm{O}$ и $\mathrm{V}_{3} \mathrm{O}$, в область более низких частот.

В то же время следует отметить, что совокупность наших результатов не подтверждает выводы авторов [8] о том, что комплекс $\mathrm{V}_{2} \mathrm{O}$ обусловливает полосу поглощения у $839 \mathrm{~cm}^{-1}$, а комплекс $\mathrm{V}_{3} \mathrm{O}$ - полосу у $884 \mathrm{~cm}^{-1}$. Эти выводы были сделаны на основе анализа (подгонки) спектров, 
измеренных при комнатной температуре для кристаллов, облученных быстрыми нейтронами. Более подробно данный вопрос будет рассмотрен в отдельной публикации.

Заключение. На основании анализа спектров ИК-поглощения, измеренных при комнатной температуре, определено положение локальных колебательных мод (ЛКМ), обусловленных валентными колебаниями узловых атомов кислорода в природном кремнии, содержащем изотопы ${ }^{28} \mathrm{Si},{ }^{29} \mathrm{Si}$ и ${ }^{30} \mathrm{Si}$. Для основной моды А-центра $\left({ }^{28} \mathrm{Si}^{16}{ }^{16} \mathrm{O}_{S^{-2}}{ }^{28} \mathrm{Si}\right)$ ЛКМ находится у $830,3 \mathrm{~cm}{ }^{-1}$, а полуширина полосы поглощения составляет $5,3 \pm 0,25 \mathrm{~cm}^{-1}$. Изотопические сдвиги ЛКМ равны $2,2 \pm 0,25 \mathrm{~cm}^{-1}$ для ${ }^{28} \mathrm{Si}^{16} \mathrm{O}_{S^{-}}{ }^{29} \mathrm{Si}$ и $4,3 \pm 0,9 \mathrm{~cm}^{-1}$ для ${ }^{28} \mathrm{Si}^{16} \mathrm{O}_{S^{-}}{ }^{30} \mathrm{Si}$ по отношению к полосе, связанной с ${ }^{28} \mathrm{Si}^{16}{ }^{16} \mathrm{O}^{-2}{ }^{28} \mathrm{Si}$.

С учетом полученных результатов изучены процессы формирования и отжига колебательных полос поглощения в облученных кислородсодержащих образцах кремния при термообработках в температурном интервале $200-400{ }^{\circ} \mathrm{C}$. Установлено, что за полосы поглощения, расположенные при комнатной температуре у 825,8 и 839,2 см$^{-1}$, ответственны комплексы дивакансиякислород и тривакансия-кислород, соответственно.

Благодарности. Работа выполнена при частичной финансовой поддержке Белорусского республиканского фонда фундаментальных исследований (проект № Ф20-111).

Acknowledgements. The work was partially supported by the Belarusian Republican Foundation for Basic Research (project no. Ф20-111).

\section{Список использованных источников}

1. Oxygen defect processes in silicon and silicon germanium / A. Chroneos [et al.] // Appl. Phys. Rev. - 2015. - Vol. 2. P. 021306 (1-15). https://doi.org/10.1063/1.4922251

2. Pajot B., Clerjaud B. Optical absorption of impurities and defects in semiconducting crystal: Defect engineering in Czochralski silicon by electron irradiation at different temperatures / J. L. Lindstrom [et al.] // Nucl. Inst. Methods Phys. Res. B. - 2002. - Vol. 186, № 1/4. - P. 121-125. https://doi.org/10.1016/s0168-583x(01)00871-0

3. Optical Absorption of Impurities and Defects in Semiconducting Crystal: Electronic Absorption of Deep Centres and Vibrational Spectra. - Berlin; Heidelberg: Springer, 2013. - 463 p.

4. Thermal double donor annihilation and oxygen precipitation at around $650{ }^{\circ} \mathrm{C}$ in Czochralski-grown Si: local vibrational mode studies / L. I. Murin [et al.] // J. Phys.: Condens. Matter. - 2005. - Vol. 17, № 22. - P. S2237-S2246. https://doi. org $/ 10.1088 / 0953-8984 / 17 / 22 / 011$

5. Коршунов, Ф. П. Радиационная технология изготовления мощных полупроводниковых приборов / Ф. П. Коршунов, Ю. В. Богатырев // Вес. Нац. акад. навук Беларусі. Сер. фіз.-тэхн. навук. - 2008. - № 4. - С. $106-114$.

6. Толкачева, Е. А. Оптические свойства и механизм образования вакансионно-кислородных комплексов $\mathrm{V}_{2} \mathrm{O}_{2}$ и $\mathrm{V}_{3} \mathrm{O}_{2}$ в облученных кристаллах кремния / Е. А. Толкачева, В. П. Маркевич, Л. И. Мурин // Физика и техника полупроводников. - 2018. - Т. 52, №. 9. - С. 973-979.

7. Толкачева, Е. А. Влияние изотопного состава природного кремния на локальные колебательные моды вакансионно-кислородных комплексов / Е. А. Толкачева, Л. И. Мурин // Журн. приклад. спектроскопии. - 2013. - Т. 80, № 4. - C. 586-590.

8. Londos, C. A. IR studies of oxygen-vacancy related defects in irradiated silicon / C. A. Londos, L. G. Fytros, G. J. Georgiou // Defect and Diffusion Forum. - 1999. - Vol. 171/172. - P. 1-32. https://doi.org/10.4028/www.scientific.net/ ddf.171-172.1

9. Pajot, B. Optical absorption of impurities and defects in semiconducting crystal: I / B. Pajot. - Berlin; Springer: Hydrogen-like centres, 2010. - $470 \mathrm{p}$.

10. The trivacancy and trivacancy-oxygen family of defects in silicon / V. P. Markevich [et al.] // Solid State Phenom. 2014. - Vol. 205/206. - P. 181-190. https://doi.org/10.4028/www.scientific.net/ssp.205-206.181

\section{References}

1. Chroneos A., Sgourou E. N., Londos C. A., Schwingenschlögl U. Oxygen defect processes in silicon and silicon germanium. Applied Physics Reviews, 2015, vol. 2, pp. 021306 (1-15). https://doi.org/10.1063/1.4922251

2. Lindström J. L., Murin L. I., Hallberg T., Markevich V. P., Svensson B. G., Kleverman M., Hermansson J. Defect engineering in Czochralski silicon by electron irradiation at different temperatures. Nuclear Instruments and Methods in Physics Research B, 2002, vol. 186, no. 1-4, pp. 121-125. https://doi.org/10.1016/s0168-583x(01)00871-0

3. Pajot B., Clerjaud B. Optical Absorption of Impurities and Defects in Semiconducting Crystal: Electronic Absorption of Deep Centres and Vibrational Spectra. Berlin, Heidelberg, Springer, 2013. 463 p.

4. Murin L. I., Lindstrom J. L., Markevich V. P., Misiuk A., Londos C. A. Thermal double donor annihilation and oxygen precipitation at around $650{ }^{\circ} \mathrm{C}$ in Czochralski-grown Si: local vibrational mode studies. Journal of Physics: Condensed Matter, 2005, vol. 17, no. 22, pp. S2237-S2246. https://doi.org/10.1088/0953-8984/17/22/011 
5. Korshunov F. P., Bogatyrev Yu. V. Radiation technology for manufacturing powerful semiconductor devices. Vestsi Natsyyanal'nai akademii navuk Belarusi. Seryya fizika-technichnych navuk = Proceedings of the National Academy of Sciences of Belarus. Physical-technical series, 2008, no. 4, pp. 106-114 (in Russian).

6. Tolkacheva E. A., Markevich V. P., Murin L. I. Optical Properties and the Mechanism of the Formation of $\mathrm{V}_{2} \mathrm{O}_{2}$ and $\mathrm{V}_{3} \mathrm{O}_{2}$ Vacancy-Oxygen Complexes in Irradiated Silicon Crystals. Semiconductors, 2018, vol. 52, no. 9, pp. 1097-1103. https:// doi.org/10.1134/s1063782618090221

7. Tolkacheva E. A., Murin L. I. Influence of the isotopic composition of natural silicon on local vibrational modes of vacancy-oxygen complexes. Journal of Applied Spectroscopy, 2013, vol. 80, no. 4, pp. 571-575. https://doi.org/10.1007/s10812013-9807-3

8. Londos C. A. IR studies of oxygen-vacancy related defects in irradiated silicon. Defect and Diffusion Forum, 1999, vol. 171-172, pp. 1-32. https://doi.org/10.4028/www.scientific.net/ddf.171-172.1

9. Pajot B. Optical Absorption of Impurities and Defects in Semiconducting Crystal: I. Berlin, Springer, Hydrogen-like centres, 2010. $470 \mathrm{p}$.

10. Markevich V. P., Peaker A. R., Hamilton B., Lastovskii S. B., Murin L. I., Coutinho J., Rayson M. J., Briddon P. R., Svensson B. G. The trivacancy and trivacancy-oxygen family of defects in silicon. Solid State Phenomena, 2014, vol. 205206, pp. 181-190. https://doi.org/10.4028/www.scientific.net/ssp.205-206.181

\section{Информация об авторах}

Толкачева Екатерина Анатольевна - кандидат физико-математических наук, старший научный сотрудник, Научно-практический центр Национальной академии наук Беларуси по материаловедению (ул. П. Бровки, 19, 220072, г. Минск, Республика Беларусь).

E-mail: talkachova@physics.by

Маркевич Владимир Павлович - кандидат физико-математических наук, старший научный сотрудник, Университет г. Манчестер (Manchester M13 9PL, United Kingdom). E-mail: V.Markevich@manchester.ac.uk

Мурин Леонид Иванович - ведущий научный сотрудник, кандидат физико-математических наук, Научно-практический центр Национальной академии наук Беларуси по материаловедению (ул. П. Бровки, 19, 220072 , г. Минск, Республика Беларусь).

\section{Information about the authors}

Ekaterina A. Tolkacheva - Ph. D. (Physics and Mathematics), Senior Researcher, Scientific and Practical Materials Research Center of the National Academy of Sciences of Belarus (19, P. Brovki Str., 220072, Minsk, Republic of Belarus).E-mail: talkachova@physics.by

Vladimir P. Markevich - Ph. D. (Physics and Mathematics), Senior Researcher, Photon Science Institute and School of Electrical and Electronic Engineering, The University of Manchester (Manchester M13 9PL, United Kingdom). E-mail: V.Markevich@manchester.ac.uk

Leonid I. Murin - Ph. D. (Physics and Mathematics), Leading Researcher, Scientific and Practical Materials Research Center of the National Academy of Sciences of Belarus (19, P. Brovki Str., 220072, Minsk, Republic of Belarus). 